\title{
Association Of Vitamin D Deficiency With Type 2 Diabetes
}

\author{
Sehrish Shafique, Rana Ansari, Hamidullah Ariff, Sajid Abbas Jafri, Naveed Faraz
}

- - - - - - - - - - - - - - - - - - - - - - - - - - - -

ABSTRACT:

Objective: To determine the association of Vitamin D deficiency with peripheral neuropathy in patients of type-2 Diabetes mellitus.

Study Design and Setting: It was a cross sectional study conducted in a private clinic in Karachi, during a period of three months.

Methodology: Total of 70 cases were included according to inclusion and exclusion criteria. Approval was sought from Ethical review committee. Consent was signed from the patients before the data collection. All the patients who were coming to the clinic, 30-75years of age, had history of type 2 diabetes for more than 5 year with HbA1c of $7 \%$ or above and had peripheral neuropathy were included. Data was collected in a pre-designed proforma. For entry of data and its statistical analysis SPSS version 20 was used.

Results: From the total 70 cases $n=41(58.5 \%)$ were vit D deficient. Most of the patients with type 2 diabetes with neuropathy were in the age group of 61-75yrs (57\%) followed by 46- 60yrs age group (30\%). This study also showed that 30 females (73\%) and 11 males $(26.8 \%)$ were deficient in vitamin D.

Conclusion: Type 2 diabetic patients with vitamin D deficiency were more at risk of developing diabetic peripheral neuropathy (DPN) earlier. It is required to supply vitamin D appropriately for preventing DPN in type 2 diabetes.

Key words: Diabetic, Neuropathy, Vitamin D, Peripheral Neuropathy

\section{INTRODUCTION:}

Type $2 \mathrm{DM}$ is among the commonly prevailing noncommunicable diseases which occurs worldwide and is of high concerns as it can cause many health-related problems. However, because of its increasing number of cases and its high burden; evidence based novel innovative approaches should be made to prevent the occurrence and risk of the disease. Recent studies showed that vitamin D is a potential diabetes risk modifier ${ }^{1}$. Diabetes is among those health care syndrome which is associated with hyperglycemia due to pathology in insulin secretion and its action on its target cell. Similarly, vitamin D deficiency has also been recognized for its global concern ${ }^{2}$. The similarity between Vitamin D

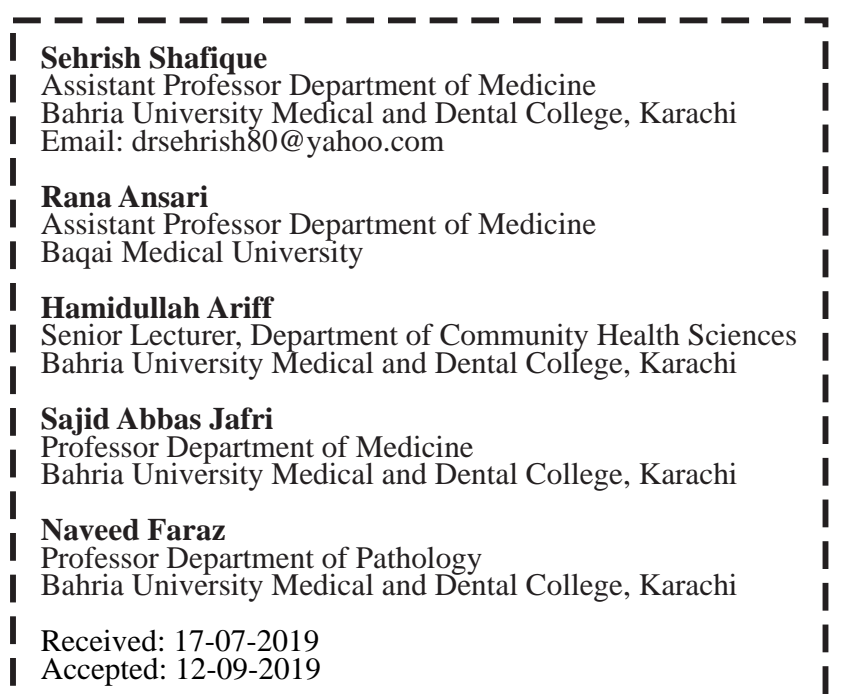

deficiency and diabetes is that both are pervasive and prevalent. According to the World Health Organization (WHO); it is estimate that by 2030; prevalence of DM rises above 366 million and the epidemic of diabetes will continue ${ }^{3}$. Vitamin D deficiency is also a commonly occurring problem in our country due to various social issues; such as lack of exposure in sunlight. About $30 \%$ to $50 \%$ of the population in the world suffers from Vitamin D deficiency. In UK, about $91 \%$ of T2DM had a Vitamin D deficiency with severe deficiency seen in $32 \%$ of patients ${ }^{4}$ Like many countries, the prevalence of vitamin D deficiency in Pakistan is affecting $53.5 \%$ of population irrespective of the age groups ${ }^{5,6,7,8}$. Studies showed that $50-90 \%$ of Pakistani population especially females are vitamin D deficient.

Vitamin D has three vital sources. The first and the most essential source of vit D is sunlight. Apart from sunlight there are also dietary sources like dairy products, cereals, milk etc. which can provide us the dietary source of vitamin D. Nutritional Supplements that contain vitamin D are considered as the third major source of vit D. So, it is extremely essential that in a human body its normal levels should be maintained for the proper functioning of the body and also for normal regulation of different body systems. Its deficiency can lead to bone pains, osteomalacia, proximal myopathies and to the extent of osteoporosis and fractures due to increase parathyroid hormone level ${ }^{9,10,11}$. According to various studies; it is suggested Vit D has an important role as an immunological suppressor of tumors, although others are less certain about $\mathrm{it}^{12}$. 
Decrease Vit $\mathrm{D}$ level is also one of the contributing factors in people for the development of both type 1 and type 2 diabetes. Beta cells which are present in the pancreas that secretes insulin also contain vitamin D receptors (VDRs) as well as the one á hydroxylase enzyme Through its effect which is regulated by calcium it has an indirect effect on causing insulin secretion. Vitamin D contributes to normalization of extracellular calcium, ensuring normal calcium flux through cell membranes and whenever there are low vitamin D levels in the body there is diminished calcium's ability to secrete insulin. If there is sufficient amount of vitamin D in the body glucose hemostasis can also be maintained in the body and thus minimizing the risk of developing diabetes. $(10,11,12)$ It plays a vital role in the plasma calcium metabolism via effects on intestinal absorption and bone metabolism.In people who have mild to moderate vitamin D insufficiency there is a risk for developing type 2 diabetes ${ }^{13}$. Not only it is associated with the occurrence of diabetes but it is also related to its complications especially with diabetic peripheral neuropathy ${ }^{14}$ as it helps in regulation and production of the neutrophic factors like neuron growth factor, neutrophin 3 and 4 and glial cell derived neutrophic factors so if it is deficient in the body there can be damaged to the neurons, and also effect on the production of neurotrophic factors which have a neuroprotective effect. Vitamin D is related to skeletal stability ${ }^{15}$ normal level of plasma vitamin D minimize the chances for the diabetes mellitus and also the progression and severity of neuropathy in high risk patients ${ }^{16,17}$ Vitamin $\mathrm{D}$ deficiency has been seen among the patients with syndrome due to specific vitamin $\mathrm{D}$ receptor gene polymorphisms ${ }^{18}$. Therefore the rationale of this study was to ascertain the frequency of 25-hydroxy vitamin $\mathrm{D}_{3}$ $\left[25(\mathrm{OH}) \mathrm{D}_{3}\right]$ deficiency and to determine their association with peripheral neuropathy in Pakistani population with type 2 diabetics.

\section{METHODOLOGY:}

All the patients who were coming to the clinic, with $30-75$ years of age, diagnosed with type 2 diabetes mellitus for more than 5 year with $\mathrm{HbA1c}$ of $7 \%$ or above with positive peripheral neuropathy which was checked through Semmes Weinstein monofilament test were included. Pregnant women, patients with history or clinical features suggesting chronic liver diseases, chronic renal disease, osteoporosis, history of any malignancies, thyroid disorders and patients already taking vitamin $\mathrm{D}$ or calcium supplements were excluded from the study. Data was collected in a pre-designed proforma.

The study was commenced after the ethical approval obtained from the ethical committee. Consent was taken from the patients prior to data collection after explaining the details of procedure. Those patients fulfilling the inclusion criteria and attending the OPD were selected. After taking their history of peripheral neuropathy Semmes Weinstein monofilament test with 5.0/10g was performed, three times at each site (dorsal between the base of digits $1-2$; ventral digits $1,3,5$; metatarsal heads $1,3,5$; medial and lateral midfoot; and heel). If the patient missed perceiving the filament more than once at one site, the test was considered abnormal at that site. If a subject did not perceive the filament at two or more of the 10 sites, the test was reported as abnormal. (19). Then all those patients with positive monofilament test, were advised to have their vitamin D levels checked in blood. Patients were labelled deficient if serum 25(OH)D concentration levels are $<20 \mathrm{ng} / \mathrm{ml}$. (1920). For data analysis SPSS version 20 were used.

\section{RESULTS:}

During the study period, a total of 70 were found to have diabetic and peripheral neuropathy among which $41(58.5 \%)$ were vit $\mathrm{D}$ deficient. The youngest being 30 years and oldest being 75 years of age. Most of the patients with type 2 diabetes with neuropathy were in the age group of $61-75 \mathrm{yrs}$ (57\%) followed by 46- 60yrs age group (30\%). Out of 41 total vit D deficiency patient ,30 were females ( $73 \%)$ and 11were males (26.8\%). (FIG.1)

Figure 1: Frequency of Vitamin D Deficiency with Neuropathy according to gender

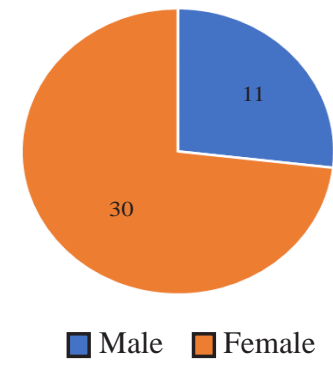

Figure 2: Distribution of vitamin D deficiency \& neuropathy patient according to age group

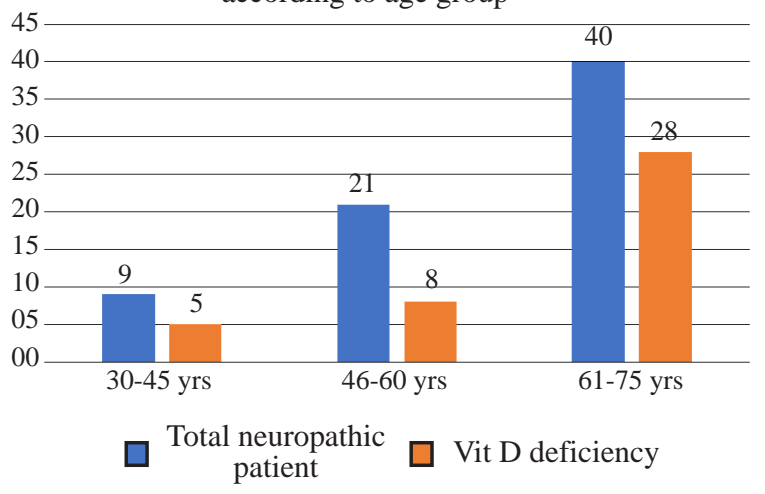

Table 1: Frequency of Vitamin D Deficiency in patients with Neuropathy

\begin{tabular}{|c|c|c|}
\hline $\begin{array}{c}\text { Total No of } \\
\text { Diabetics with } \\
\text { Neuropathy }\end{array}$ & $\begin{array}{c}\text { Vitamin D } \\
\text { Deficiency in } \\
\text { Diabetics with } \\
\text { Neuropathy }\end{array}$ & Percentage \% \\
\hline 70 & 41 & $58.5 \%$ \\
\hline
\end{tabular}




\section{DISCUSSION:}

Diabetes is one of those diseases if it is not controlled, can lead to devastating microvascular complications such as eye, kidneys and brain which can compromise the quality of life and can also lead to high morbidity and mortality. This study determine the levels of 25-hydroxy vitamin $\mathrm{D}_{3}$ among patient with neuropathy especially in diabetic patient, and to determine the correlation between low $25(\mathrm{OH}) \mathrm{D}_{3}$ levels with peripheral neuropathy, because vitamin D has a neuroprotective effect and it can prevent the progression and severity of neuropathy. There are numerous factors that can cause the development of peripheral neuropathy in diabetes like inflammation, oxidative stress causes damage to blood vessels and further can lead to nerve ischemia, enhance advanced glycation end products all can lead to the development of neuropathy in the peripheral nerves ${ }^{21}$. Past studies have been shown that diabetic patients are almost twice more prone to have vitamin D deficiency as compared to normal healthy patient. So, in diabetics sufficient levels of vit $\mathrm{D}$ has a therapeutic role in the prevention but also in decreasing the severity and progression of illnesss ${ }^{22}$. In our study also, it was observed that out of total 70 type 2 diabetes mellitus with peripheral neuropathy among which $41(58.5 \%)$ were found to have vitamin D deficiency(table 1) In this study the association seen between peripheral neuropathy and vitamin D deficiency, and these results were in agreement also with the results of the studies in which $67 \%$ of patients with diabetes have diabetic peripheral neuropathy with vit D deficiency levels, <20 ng,. Multiple studies have also found that serum vitamin $\mathrm{D}$ level in patients with diabetic peripheral neuropathy is significantly lower than those in healthy persons ${ }^{23,24}$. previous studies also showed that symptoms of nerves dysfunction can be prevented by correcting VIT D deficiency. Prevalence of Vit D deficiency is mostly associated with distal symmetric polyneuropathy. Neurological pain minimized by doing vit D deficiency corrected ${ }^{24}$. There are multiple factors which can lead to vitamin D deficiency like poor socioeconomic status, lack of proper care on diet, poor exposure to sunlight due to traditional dress all can be the contributing factors for deficiency in our country. The results shown in our study were also consistent with the other studies which have been done in Pakistan and Saudi Arabia to determine the frequency of vitamin D deficiency in diabetics ${ }^{24,25}$. Although, the studies which were conducted in western population have showed high prevalence of vit $\mathrm{D}$ deficiency that was $76 \%$ in diabetics. Previous studies shows incidence rate of vit D deficiency equal to $60.5 \%$ in Switzerland, Australia (43\%), Italy(25\%), and North America(15\%). Our finding were consistent with previous studies as well. Also past studies revealed that the level of vitamin $\mathrm{D}$ in diabetics are lower than non- diabetic individuals ${ }^{26,27,28}$ in one of study done in our country shows that $53.5 \%$ had deficient levels.
In this study the patients were categorized into three age groups Relative frequency of decrease Vit D was found to be higher in old age group $57 \%$ (figure 2) which were also consistent with the previous studies ${ }^{25}$. Patients with increasing age were more at risk to develops vitamin D deficiency as a result of many reasons: like decreased in the number of vitamin D receptor, decrease calcium absorption in response to circulating $1,25(\mathrm{OH}) 2 \mathrm{D}$, decreased dermatological metabolism of vitamin $\mathrm{D}$, and above all as with age so many organ compromise their function so as a result less production of active form of vit $\mathrm{D}(1,25(\mathrm{OH}) 2 \mathrm{D})$ by the kidneys ${ }^{29}$.

In our study there were more females $(73 \%)$ as compared to males (27\%) (fig 1) and this result consistent with the past studies which shows high incidence of vit D deficiency correlated with gender differences with deficiency more in females then males ${ }^{25}$. Our study finding of diabetic female with neuropathy had significantly lowered $25(\mathrm{OH})$ level were seen in past studies as well but one of the study in Iran shows no significant association between vitamin D levels and gender differences ${ }^{30}$. Therefore, it was recommended that vitamin $\mathrm{D}$ level of diabetic monitored regularly to prevent the complications of neuropathy like foot infections, ulcerations that can lead to foot amputation can be minimized and hence can reduce their morbidity and mortality.

Smaller sample crossectional nature of the study design were the limitation of the study

\section{CONCLUSION:}

Type 2 diabetic patients with vit d deficiency were at more risk of developing earlier peripheral neuropathy. Diabetic female were more susceptible of having peripheral neuropathy due to vit $\mathrm{D}$ deficiency as compare to male.

\section{REFERENCES:}

1. Miñambres I, Sánchez-Quesada JL, Vinagre I, SánchezHernández J, Urgell E, de Leiva A, et al. Hypovitaminosis D in type 2 diabetes: relation with features of the metabolic syndrome and glycemic control. Endocrine Research. 2015; 40(3): 160-5.

2. Kostoglou-Athanassiou I, Athanassiou P, Gkountouvas A, Kaldrymides P. Vitamin D and glycemic control in diabetes mellitus type 2. Therapeutic Advances in Endocrinology and Metabolism. 2013; 4: 122-8.

3. Teresa Martin, RD, CDE, LD, and R. Keith Campbell, RPh, CDE, FASHP. Vitamin D and Diabetes. Diabetes Spectrum 2011;24(2):113-18.

4. Rahman S, Majumder AA. Links between Vitamin D deficiency and diabetes associated macro vasculopathy. South East Asia Journal of Public Health 2015;5(2):4-6.

5. Sheikh A, Saeed Z, Jafri SA, Yazdani I, Hussain SA. Vitamin $\mathrm{D}$ levels in asymptomatic adults- a population survey in Karachi, Pakistan. PloS One 2012;7(3):e33452.

6. Akhtar S. The prevalence and correlates of vitamin D deficiency-perspectives from Pakistan. Pakistan Journal Pharmaceutical Sciences 2016;29(4):1325-30. 
7. Akhtar S. Vitamin D status in South Asian populations - risks and opportunities. Journal of Critical Review in Food Science and Nutrition 2016;56(11):1925-40.

8. Masood SH, Iqbal MP. Prevalence of vitamin D deficiency in South Asia. Pakistan Journal of Medical Sciences. 2008;24(6):891-97.

9. Mezza T, Muscogiuri G, Sorice GP, Prioletta A, Salomone E, Pontecorvi A, Giaccari A. Vitamin D Deficiency: A New Risk Factor for Type 2 Diabetes? Annals of Nutrition and Metabolism 2012;61(4):337-48.

10. Riaz H, Finlayson AE, Bashir S, Hussain S, Mahmood S, Malik F, Godman B. Prevalence of Vitamin D deficiency in Pakistan; implications for the future. Journal of Expert Review of Clinical Pharmacology 2016;9(2):329-38.

11. Khan H, S, OH, Chowdhury R. Vitamin D, type 2 diabetes and other metabolic outcomes: a systematic review and metaanalysis of prospective studies. The Proceedings of the Nutrition Society. 2013;72(1):89-97.

12. Afzal S, Bojesen SE, Nordestgaard BG. Low 25-hydroxyvitamin D and risk of type 2 diabetes: a prospective cohort study and metanalysis. Journal of Clinical Chemistry. 2013;59(2): 381-91.

13. Tsur A, Feldman BS, Feldhammer I, Hoshen, M.B, Leibowitz, G., Balicer, R.D. Decreased serum concentrations of 25hydroxycholecalciferol are associated with increased risk of progression to impaired fasting glucose and diabetes. Diabetes Care 2013 ;36(5): 1361-67.

14. Pittas, AG, Lau J, Hu FB et al. The role of vitamin D and calcium in type 2 diabetes. A systematic review and metaanalysis. Journal of Clinical Endocrinology and Metabolism.2007; 92(6):2017-29.

15. Seham E. Abdelsadek, Entesar O. El Saghier and Sabah I. Abdel Raheem.Serum 25(OH) vitamin D level and its relation to diabetic peripheral neuropathy in Egyptian patients with type 2 diabetes mellitus. The Egyptian Journal of Neurology, Psychiatry and Neurosurgery 2018. https://doi.org/10.1186 /s41983-018-0036-9

16. Holick MF, Binkley NC, Bischoff-Ferrari HA, Gordon CM , Hanley DA, Heaney RP, Murad MH, Weaver CM. Evaluation, treatment anprevention of vitamin D deficiency: an Endocrine Society clinical practice guideline. Journal of Clinical Endocrinology Metabolism. 2011;96(7):1911-30

17. Pittas AG, Nelson J, Mitri J, Hillmann W, Garganta C, Nathan DM, et al. Plasma 25hydroxyvitamin D and progression to diabetes in patients at risk for diabetes: an ancillary analysis in the Diabetes Prevention Program. Diabetes care. 2012;35(3):565-73.

18. Guang-Bo Qu, Ling-Ling Wang, Xue Tang, Wei Wu, YeHuan Sun GB. Qu et al. The association between vitamin D level and diabetic peripheral neuropathy in patients with type 2 diabetes mellitus: An update systematic review and metaanalysis. The Journal of Clinical \& Translational Endocrinology 2017;(9): 25-31.
19. Qi Zhang, Na Yi, Siying Liu,Hangping Zheng Xiaona Qiao ,Qian Xiong, Xiaoxia Liu, Shuo Zhang, Jie Wen .Easier operation and similar power of $10 \mathrm{~g}$ monofilament test for screening diabetic peripheral neuropathy. Journal of International Medical Research 2018; 46(8): 3278-84.

20. Holick MF. Vitamin D deficiency. The New England Journal of Medicine NEJM. 2007;357(3):266-81

21. Kayaniyil S, Retnakaran R, Harris SB et al. Prospective associations of vitamin $\mathrm{D}$ with â-cell function and glycemia. the Prospective Metabolism and Islet cell Evaluation (PROMISE) cohort study. Diabetes 2011;60(11):2947-53.

22. Shehab D, Al-Jarallah K, Mojiminiyi OA, Al Mohamedy H,Abdella NA. Does Vitamin D deficiency play a role in peripheral neuropathy in Type 2 diabetes. 2012; 29(1):43-9.

23. Almetwazi SM, Noor AO, Almasri DM, Popovici L, Alhawassi T, Albertian KA, Harrington CA. The association of vitamin D deficiency and glucose control among diabetic patients. Saudi Pharmaceutical Journal 2017;25(8):1179-83.

24. Ahmadieh H, Azar ST, Lakkis N, Arabi A. Hypovitaminosis $\mathrm{D}$ in patients with type 2 diabetes mellites' relation to disease control and complications. ISRN Endocrinology Oct 2013:641098. doi: 10.1155/2013/641098. eCollection 2013

25. Usluogullari CA, Balkan F, Caner S, Ucler R, Cafer Kaya C, Ersoy R and Cakir B. The relationship between microvascular complications and vitamin D deficiency in type 2 diabetes mellitus. BMC Endocrine Disorders 2015 ;9(15):33

26. Al-Agha AE, Ahmad IA. Association among Vitamin D Deficiency, Type 1 Diabetes Mellitus and Glycemic Control. Journal of Diabetes and Metabolism 2015; 6:9. DOI: 10.4172/2155-6156.1000594

27. Gagnon C, Lu ZX, Magliano DJ, et al. Serum 25 hydroxy vitamin $\mathrm{D}$, calcium intake, and risk of type 2 diabetes after 5 years: results from a national, population based prospective study (the Australian Diabetes, Obesity and Lifestyle study). Diabetes Care 2011; 34 (5): 1133-8.

28. Mohammed I. Oraby, Mona Abu Srie, Sanaa Abdelshafy, Eman Elfar Diabetic peripheral neuropathy: the potential role of vitamin D deficiency. The Egyptian journal of Neurology, Psychiatry and Neurosurgery 2019 55:10.

29. Sarita Bajaj, raj pratab Singh, NC Dwivedi .Vitamin D levels and microvascular complications in type 2 diabetes .olism. 2014;18(4): 537-41.

30. Jadoon SA, Ahmed A, Alam MA. VITAMIN D DEFICIENCY IN PAKISTAN: TIP OF ICEBERG. Journal of Ayub Medical College Abbottabad: JAMC 2018;30(1):78-80. 